\title{
Yeast as a versatile system for heterologous protein expression; genetically modified yeast strain to express human drug metabolism enzymes
}

Keywords: Yeast, recombinant proteins, mammalian cell, genetic manipulation, saccharomyces cerevisiae, cytochrome $\mathrm{p} 450$

\section{Introduction}

A wide variety of recombinant expression systems are available today to produce large amounts of recombinant proteins. Choosing the right expression system for a particular application is the key to success, whether it is a prokaryotic system, viral system, systems mimicking eukaryotic expression systems like yeast, insect or mammalian cell line expression system. Model organisms are often chosen on the basis that they are amenable to experimental manipulation. This usually includes characteristics such as generation time, cell culture complexity, accessibility, genetic manipulation, conservation of mechanisms, expression level, extracellular expression, post-translational modification \& processing, scale-up and potential economic benefit.

There are a lot of factors which affect the overall yield of any recombinant protein in any organism. One of the most important parts for the system to be successful is the expression plasmid/vector used for the expression of a heterologous gene.

Another important factor required for desired expression of a heterologous gene is the choice of correct host system as explained above. Mammalian cells as hosts for heterologous expression is best for the expression of human genes but unfortunately this is associated with low yields, high cost and long generation times. On the other hand prokaryotic system, although results in high protein yields in considerably less time but lacks the post-translational modifications and processing required for most human proteins.

Yeast (Saccharomyces cerevisiae) has been the best choice of a eukaryotic system as being a single-celled eukaryote, has proven to be more like human cells in its molecular structure and function than anyone imagined. The cell cycle in yeast is very similar to the cell cycle in humans, and regulated by homologous proteins. It is less expensive to scale up when compared over other more complex systems like insects and mammalian cells that exhibit slow growth. Additional features include high expression and the ability to perform post translational modifications as compared to E. coli based systems.

One of the common concerns for effective high protein production and purification in any host is the proteolytic degradation of the recombinant gene products by host-specific proteases. ${ }^{1}$ De novo proteins synthesis in any system is a continuous cycle of synthesis and degradation over time (in situ protease dependent degradation/ cleavage), based on the requirement of that particular cellular system. Proteolysis can occur during expression or during purification. A vast number of research publications and literature reviews have demonstrated that the protein degradation can be decreased by deleting the major protease producing genes. "Proteases" refer to a group of enzymes whose catalytic function is to breakdown proteins,
Volume 5 Issue I - 2017

Rajeev Soni

Independent Biotech Consultant, India

\begin{abstract}
Correspondence: Rajeev Soni, Independent Biotech Consultant, I74 DSR Sunrise Towers, Channasandra Road,Whitefield, Bangalore -560066, Karnataka, India, Tel +919916725500, Email sonicantab@gmail.com
\end{abstract}

Received: April 22, 2017 | Published: July 06, 2017

by hydrolysis of the peptide bonds. Consequently, production of many proteins, particularly heterologous expressed proteins by use of strains that are deficient in proteases can significantly improve overall yields. ${ }^{2}$ These findings demonstrate that construction of a proteasedeficient host system is useful in effective protein production and purification of any recombinant protein.

Heterologous expression of cytochrome P450s has been established in several organisms. Saccharomyces cerevisiae was used as the first host for the heterologous expression of mammalian cytochrome P450. ${ }^{3}$ Although various P450 isozymes have been successfully expressed in $S$. cerevisiae, their expression levels fluctuated. ${ }^{4}$ Review and US Patents namely US 5635369 and US 6579693) and some P450 isozymes, for unknown reasons, were not expressed in $S$. cerevisiae. It has been reported that the expression efficiency of P450s in $S$. cerevisiae can be improved by appropriate alteration of the yeast strain or the expression plasmid. ${ }^{4,5}$

This review describes the strategy for making the proteasedeficient haploid yeast strain by sequential disruption of major protease encoding genes of $S$. cerevisiae and further modification of yeast genome, to be used for the expression of human drug metabolism enzymes. This can be achieved by the integration of yeast sequence biased human cytochrome P450 reductase gene into the proteasedeficient yeast strain, PDYS, to be used for high level expression of catalytically active cytochrome P450s.

The protease deficient strain (PDYS) is constructed by site specific homologous recombination. An haploid yeast strain of mating type alpha is chosen that has enough and relevant auxotrophic markers, wherein the genes to be disrupted for making protease deficient strain are protease $\mathrm{A}$ and protease $\mathrm{B}$ coding genes. Both genes are disrupted sequentially resulting in the PDYS strain.

The PDYS strain developed by sequential disruption of protease $\mathrm{A}$ and $\mathrm{B}$ genes makes the strain amenable to producing heterologous proteins in a cost effective and economical manner.

The PDYS strain can be further modified by integration of yeast sequence biased human cytochrome P450 reductase (plasmid pYRI 
OR) for high level expression of catalytically active cytochrome P450s (Figure 1A).

Cytochrome P450 (P450s) is a super family of haemoproteins that catalyse the oxidation of a wide variety of endogenous and xenobiotic chemicals, including therapeutic drugs and carcinogens. $\mathrm{P} 450$ s have been found in all tissues but are localized mainly in the liver. P450-dependent metabolism requires two protein components, P450, the catalytic partner and NADPH-P450 oxido reductase (human cytochrome P450 reducatse), the regulatory partner. Both enzymes are embedded in the membrane of endoplasmic reticulum, and cytochrome P450 reductase shuttles electrons from NADPH to P450 for its functional activity.

$$
\mathrm{NADPH}+\mathrm{H}^{+}+\mathrm{O} 2+\mathrm{RH}==>\mathrm{NADP}^{+}+\mathrm{H}_{2} \mathrm{O}+\mathrm{R}-\mathrm{OH}
$$

The role of $\mathrm{P} 450$ s in drug metabolism has made the $\mathrm{P} 450$ enzyme system an important tool in drug development process. The base yeast strain is genetically engineered to express the yeast sequence biased human cytochrome P450 reductase under the galactose inducible promoter from the single integrated copy in the yeast genome. The yeast sequence biased catalytic partner of specific human human cytochrome P450s are cloned individually on to episomal plasmids (pYRE CYP) and transformed into this base strain to express the catalytically active human cytochrome P450s (Figure 1B).

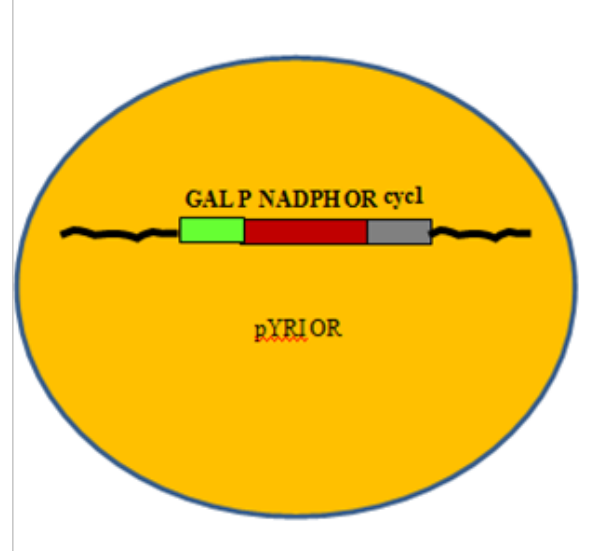

Figure IA

\section{Acknowledgements}

None.

\section{Conflict of interest}

The author declares no conflict of interest.

\section{References}

1. Alimjan Idiris, Kewei Bi, Hideki Tohda, et al. Construction of a proteasedeficient strain set for the fission yeast Schizosaccharomyces pombe, useful for effective production of protease-sensitive heterologous proteins. Yeast. 2006;23(2):83-99.

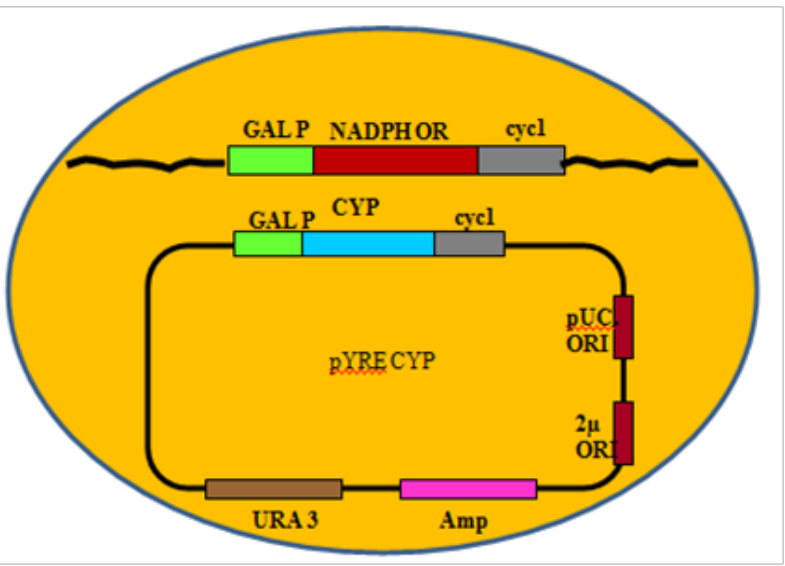

Figure IB

2. Martin AGG, Christopher EW, David PM, et al. Generation of ProteaseDeficient Strains and Their Use in Heterologous Protein Expression. Methods in Molecular Biology. 1998;103:81-94.

3. Oeda K, Sakaki T, Ohkawa H. Expression of rat liver cytochrome $\mathrm{P}-450 \mathrm{MC}$ cDNA in Saccharomyces cerevisiae. DNA. 1985;4(3):203210 .

4. Gonzalez FJ, Korzekwa KR. Cytochromes P450 expression systems Annu Rev Pharmacol Toxicol. 1995;35:369-390.

5. Urban P, Cullin C, Pompon D. Maximizing the expression of mammalian cytochrome P-450 monooxygenase activities in yeast cells. Biochimie. $1990 ; 72(6-7): 463-472$ 